\title{
Cumplimiento de profilaxis antibiótica en un Servicio de Urología
}

\author{
Ballestero Diego R, Rebollo Rodrigo H, Gutiérrez Baños JL, Portillo Martín JA, \\ Zubillaga Guerrero S, Ramos Barseló E.
}

Hospital Universitario Marqués de Valdecilla. Santander.

Actas Urol Esp. 2008;32(4):446-454

\begin{abstract}
RESUMEN
CUMPLIMIENTO DE PROFILAXIS ANTIBIÓTICA EN UN SERVICIO DE UROLOGÍA

Introducción: La tasa de infección del sitio quirúrgico es un buen indicador de la calidad asistencial en los servicios quirúrgicos. La profilaxis antibiótica representa una medida de eficacia probada en la prevención de la infección del sitio quirúrgico y su cumplimiento constituye un objetivo en los contratos de gestión de los servicios de salud. La eficacia de esta medida preventiva requiere la existencia de protocolos de actuación actualizados y la evaluación de los mismos.

Objetivo: Evaluar el cumplimiento de ese protocolo antibiótico en nuestro Servicio analizando las causas de profilaxis inadecuada de forma global y por procedimientos.

Material y métodos: El servicio de Medicina Preventiva, como observador externo, por medio de un estudio retrospectivo analizó 695 intervenciones quirúrgicas (en pacientes ingresados y ambulantes) realizadas por el servicio de Urología durante los años 2003 al 2006 para su evaluación anual del cumplimiento del protocolo de profilaxis antibiótica.

Se considera profilaxis inadecuada la administrada no estando indicada, la no administrada estando indicada y la administrada con pauta incorrecta.

Resultados: El cumplimiento del protocolo es adecuado en el 83,16\% de las intervenciones. La causa de inadecuación más frecuente fue la pauta incorrecta en el 15,3\%. Dentro de esta pauta incorrecta la causa principal fue el momento de administración tardío del antibiótico profiláctico y en menor medida una duración incorrecta de la pauta antibiótica.

Conclusiones: El porcentaje de cumplimento del protocolo antibiótico en nuestro servicio de Urología es alto.

La evaluación detectó dos problemas de diferente índole y solución: El adecuar el momento de administración del antibiótico es un problema funcional, estructural y organizativo a resolver junto al servicio de Anestesiología; El número de dosis de antibiótico en cirugía abierta de próstata requiere el conocimiento y cumplimiento estricto de la pauta por parte de los cirujanos

La efectividad de la profilaxis antibiótica requiere la existencia de protocolos adecuados y actualizados para la unificación de criterios entre los profesionales, para detectar nuevos problemas, así como para buscar soluciones para un correcto cumplimiento del mismo.

Palabras clave: Profilaxis antibiótica. Cirugía. Infección.
\end{abstract}

\section{ABSTRACT}

PERFORMANCE OF ANTIBIOTIC PROPHYLAXIS IN OUR UROLOGIC DEPARTMENT

Introduction: The rate of surgical site infection is a good indicator of the quality of care in surgical departments. Antibiotic prophylaxis represents a measure of proven efficiency in preventing the infection in a surgical site, and its fulfilment is a main goal in management contracts of health services. The efficiency of this preventive measure requires the existence of updated protocols and performance evaluation.

Objective: To evaluate the compliance of this antibiotic protocol in our Urology Department with the global analysis of the causes of inadequate prophylaxis and by procedures.

Matherial and Methods: The Department of Preventive Medicine as an outside observer, through a retrospective study, analyzed 695 urological surgical procedures (inpatient and outpatient) during the years 2003 and 2006 for its annual assessment of compliance with the antibiotic prophylaxis protocol. Administration of non-indicated prophylaxis, non-administration of indicated prophylaxis and incorrect dosage are considered as inappropriate prophylaxis.

Results: The compliance of the protocol was appropriate in $83.16 \%$ of the operations. The cause of the most frequent inadequacy was an incorrect pattern in $15.3 \%$. Within this incorrect pattern the main reason was the delay of administration of prophylactic antibiotic and to a lesser extent an incorrect length of the antibiotic regime

Conclusions: The percentage of compliance with the antibiotic protocol in our Urology Department is high. The evaluation detected two problems of a different nature and solution: to adapt the timing of antibiotics is a functional, structural and organizational problem to be resolved in accordance with the Anaesthesiology Department. The number of doses of antibiotics in open prostate surgery requires knowledge and strict adherence to the guidelines by the surgeons.

The efficiency of antibiotic prophylaxis requires the existence of appropriated and updated protocols for the unifications of criteria among professionals to detect new problems as well as to find solutions for its adequate compliance.

Keywords: Antibiotic prophylasis. Surgery. Infection. 
$\mathrm{L}_{\mathrm{s}}^{\mathrm{a}}$ a infección nosocomial (IN) y la infección del sitio quirúrgico (IQ) influyen de forma importante en la morbilidad, en las complicaciones y en el bienestar del paciente. Este aumento de morbi-mortalidad y complicaciones producen un incremento en la estancia media hospitalaria, así como un aumento de los costes originados para el sistema nacional de salud, por lo que representa un importante problema socio-sanitario descrito en numerosos trabajos científicos ${ }^{1-7}$.

Las tasas de IQ, especialmente en cirugía limpia, se consideran un indicador de resultado fiable de la calidad asistencial del trabajo realizado y permiten comparaciones internas a lo largo del tiempo y externas con otros hospitales.

En el contexto actual, la seguridad del paciente es un objetivo adoptado y difundido por múltiples organizaciones sanitarias, por lo que conocer la tasa de IQ y sobre todo promover acciones de mejora y de eficacia probada para intentar disminuirlas es un compromiso ineludible para la gestión de un servicio quirúrgico ${ }^{8,9}$. En estos momentos, la Agencia de Calidad del Ministerio de Sanidad, en los convenios firmados con las comunidades autónomas para priorizar la seguridad del paciente en las estrategias de salud tiene entre sus objetivos la prevención de la infección quirúrgica en los hospitales ${ }^{9}$.

La profilaxis antimicrobiana representa una medida de eficacia probada en la prevención de estas infecciones, incluyendo la cirugía urológica ${ }^{10-13}$. Esto supone la implantación de un protocolo antibiótico ajustado a nuestros procedimientos, infecciones y etiología microbiana, datos que se obtienen del sistema prospectivo de vigilancia y control de IN que llevan a cabo el Servicio de Medicina Preventiva7.

El cumplimiento de esta profilaxis constituye un objetivo en los contratos de gestión de los servicios de salud, considerándose uno de los indicadores de calidad asistencial. La eficacia de esta medida preventiva requiere la existencia de protocolos de actuación que se actualicen y se evalúen de forma periódica $^{14}$.

En nuestro servicio se utiliza desde hace años un protocolo de PA aprobado y revisado periódicamente por la Comisión de infecciones del hospital. El servicio de Medicina Preventiva y Seguridad del paciente evalúa de forma periódica el cumplimiento de este protocolo, así como las tasas de IQ de los diferentes procedimientos quirúrgicos. El propósito de nuestro estudio ha sido evaluar nuestro protocolo antibiótico, así como el cumplimiento del mismo aplicado en nuestro servicio analizando las causas de profilaxis inadecuada de forma global y por procedimientos.

\section{MATERIAL Y MÉTODOS}

Nuestro ámbito de estudio es un servicio de urología de un hospital de tercer nivel con una media diaria de 30 camas, unos ingresos anuales en torno a 860 pacientes y de unas 530 intervenciones realizadas al año.

El servicio de Medicina Preventiva es el responsable de la vigilancia de la infección y el evaluador externo del cumplimiento del protocolo de PA. Por medio de una muestra retrospectiva representativa se analizaron 695 intervenciones quirúrgicas (en pacientes ingresados y ambulantes) realizadas en el servicio de Urología durante diversos periodos de los años 2003 al 2006. Se incluyeron todas las cirugías abiertas y endoscópicas, de carácter urgente y programado, a excepción del trasplante renal.

Se revisaron todas las historias clínicas de los pacientes intervenidos en Urología, ingresados o no (cirugía menor ambulatoria) en un determinado periodo de tiempo, valorando la correcta o incorrecta aplicación del protocolo antibiótico vigente. La amplitud de este periodo de tiempo fue calculada de forma que el número de casos se encuentre por encima del tamaño muestral previamente calculado, necesario para estimar el cumplimiento anual del protocolo.

El periodo de recogida de datos se estableció en 2 meses en 2003 y en 1,5 meses en los años 20042006.

Los fármacos y las dosis empleadas en esta profilaxis, que han permanecido invariables durante los 4 años de estudio, se reflejan en la Tabla 1. Es de señalar que el establecimiento de estas pautas se realiza en función de los gérmenes aislados en cada tipo de cirugía y de sus resistencias frente a los antimicrobianos ${ }^{15}$. Estos protocolos han sido aprobados y revisados por la Comisión de Infecciones y de Profilaxis antibiótica del hospital. Los datos obtenidos se han expresado en porcentaje de adhesión al protocolo.

Consideramos profilaxis adecuada la profilaxis administrada estando indicada y con pauta correcta, así como la profilaxis no administrada no estando indicada. 
Tabla 1. Protocolo antibiótico de nuestro servicio.

\section{PROFILAXIS QUIRÚRGICA EN UROLOGÍA}

1. CIRUGía ENDOSCóPICA (resección transuretral de próstata y vejiga, uretrotomía endoscópica, ureterorrenoscopia intervencionista y nefroscopia percutánea): AMPICILINA (1 dosis) + GENTAMICINA (1 dosis)

2. CIRUGía ABIERTA

a. Intervenciones de próstata y vejiga abiertas en pacientes portadores de sondaje vesical: AMPICILINA (4 dosis) + GENTAMICINA (1 dosis).

b. Intervenciones de próstata y vejiga abiertas en pacientes NO portadores de sondaje vesical, nefrectomías y cirugia genital: AMOXICILINA-CLAVULÁNICO (1 dosis)

Dosis: 2 givia: IV

Administración: en el momento de comenzar la inducción anestésica

En alérgico a b-lactámicos: GENTAMICINA (1 dosis)

* Nota sobre dosificación de:

\section{AMPICILINA}

Dosis: $2 \mathrm{~g} \quad$ Vía: IV Pauta: 2 g cada 6 horas durante 24 horas

Administración: en el momento de comenzar la inducción anestésica

\section{GENTAMICINA}

Dosis: $240 \mathrm{mg}$. Observaciones: en caso de paciente menor de $60 \mathrm{~kg}$ o mayor de $100 \mathrm{~kg}$ de peso, se ajustará de forma que la dosis administrada no exceda de $4 \mathrm{mg} / \mathrm{kg} / \mathrm{día}$

Vía: IV

Administración: en el momento de comenzar la inducción anestésica

3. CIRUGÍA ABIERTA DE VEJIGA Y RECONSTRUCCION CON ASA INTESTINAL (enterocistoplastia de sustitución): AMPICILINA + GENTAMICINA + CLINDAMICINA

AMPICILINA (20 dosis)

Dosis: $1 \mathrm{~g} \quad$ Vía: IV Pauta: $1 \mathrm{~g}$ cada 6 horas durante 5 días

Administración: en el momento de comenzar la inducción anestésica

GENTAMICINA (5 dosis)

Dosis: $240 \mathrm{mg}$. Observaciones: en caso de paciente menor de $60 \mathrm{~kg}$ o mayor de $100 \mathrm{~kg}$ de peso, se ajustará de forma que la dosis administrada no exceda de $4 \mathrm{mg} / \mathrm{kg} / \mathrm{dí}$.

Vía: IV Pauta: $240 \mathrm{mg}$ cada 24 horas durante 5 días.

Administración: en el momento de comenzar la inducción anestésica

CLINDAMICINA (20 dosis)

Dosis: $600 \mathrm{mg} \quad$ Vía: IV Pauta: $600 \mathrm{mg}$ cada 6 horas durante 5 días

Administración: en el momento de comenzar la inducción anestésica

4. PRÓTESIS DE PENE: VANCOMICINA + GENTAMICINA

VANCOMICINA (3 dosis)

Dosis: $1 \mathrm{~g} \quad$ Vía: IV Pauta: $1 \mathrm{~g}$ cada 8 horas

Administración: en el momento de comenzar la inducción anestésica

GENTAMICINA ( 1 dosis)

Dosis: $240 \mathrm{mg}$. Observaciones: en caso de paciente menor de $60 \mathrm{~kg}$ o mayor de $100 \mathrm{~kg}$ de peso, se ajustará de forma que la dosis administrada no exceda de $4 \mathrm{mg} / \mathrm{kg} /$ día

Vía: IV

Administración: en el momento de comenzar la inducción anestésica

\section{BIOPSIA DE PRÓSTATA TRANSRECTAL: CIPROFLOXACINO}

Limpieza mecánica de ampolla rectal por medio de enema.

CIPROFLOXACINO (2 dosis)

Dosis: $500 \mathrm{mg}$ Vía: oral

Administración: 12 horas antes de la biopsia y la segunda dosis 12 horas después de la realización de la biopsia

\section{CIRUGÍA GENITAL MENOR (Circuncisión, vasectomía, frenillo)}


La profilaxis inadecuada consiste en la administrada no estando indicada, la no administrada estando indicada y la administrada con pauta incorrecta.

Dentro del último grupo señalado de profilaxis administrada con pauta incorrecta puede ser por tres causas no excluyentes: elección de antibiótico diferente al protocolizado, administración precoz o tardía del mismo y diferente número de dosis del que figura en el protocolo.

El diagnóstico de infección nosocomial y concretamente de infección quirúrgica, se realizó en función de los criterios definidos del estudio EPINE (adaptación de los criterios del Centers for Disease Control) ${ }^{1,16}$.

Además de la difusión (mensual de la IN y anual del cumplimiento del protocolo de PA) se ha realizado al menos una reunión anual donde todos los miembros del servicio analizan los datos obtenidos y se establecen acciones de mejora o propuestas de cambios, tanto del sistema de vigilancia como de los protocolos y su evaluación.

\section{RESULTADOS}

Analizando los resultados de forma global (Tabla 2), observamos que la tasa de profilaxis adecuada alcanza un $83,16 \%$, oscilando entre el $71,71 \%$ del 2003 y el 88,29\% del 2004.
Por el contrario la profilaxis inadecuada se establece en una tasa media de $16,83 \%$ en el periodo descrito. Dentro de la profilaxis inadecuada observamos que la situación más frecuente es la administración de la profilaxis con pauta incorrecta, con tasas que superan el $80 \%$ frente al resto de situaciones.

Profundizando en las razones de esta administración con pauta incorrecta (Tabla 3), se puede observar que en la mayoría de los casos se debe a un momento de inicio de la administración incorrecto, con porcentajes entre el 50 y el 92\%, señalando únicamente un $35 \%$ de duración incorrecta de la pauta de profilaxis en el 2004.

Esta inadecuación con respecto al momento de administración, se debe en la mayoría de los casos a la administración tardía del antibiótico elegido, en la cual se realizaba tras realizar todas las maniobras anestésicas una vez empezada la intervención, y no en la inducción anestésica como indica el protocolo.

La duración incorrecta de la pauta antibiótica está en relación a 2 situaciones: la falta de administración de las 3 dosis postoperatorias de ampicilina en la cirugía abierta de próstata y la administración de profilaxis prolongada en las nefrectomías con amoxicilina-clavulánico. La elección del antibiótico, supone

Tabla 2. Cumplimiento del protocolo de profilaxis de forma global

\begin{tabular}{|c|c|c|c|c|c|c|}
\hline \multirow[b]{2}{*}{ Año 1} & \multirow[b]{2}{*}{$\begin{array}{l}\text { Total infección } \\
\text { quirúrgica }\end{array}$} & \multirow[b]{2}{*}{$\begin{array}{c}\text { Profilaxis } \\
\text { adecuada } \\
\text { n (\%) }\end{array}$} & \multicolumn{4}{|c|}{ Profilaxis inadecuada } \\
\hline & & & $\begin{array}{c}\text { Administrada } \\
\text { no indicada } \\
\text { n (\%) }\end{array}$ & $\begin{array}{c}\text { No administrada } \\
\text { indicada } \\
\mathbf{n}(\%)\end{array}$ & $\begin{array}{c}\text { Administrada } \\
\text { incorrecta } \\
\mathbf{n}(\%)\end{array}$ & $\begin{array}{c}\text { Total } \\
\text { inadecuada } \\
\mathbf{n}(\%)\end{array}$ \\
\hline 2003 & 152 & $109(71,71)$ & $4(9,30)$ & $0(0)$ & $39(90,70)$ & 28,29 \\
\hline 2004 & 205 & $181(88,29)$ & $4(16,67)$ & $0(0)$ & $20(83,33)$ & 11,71 \\
\hline 2005 & 148 & $127(85,81)$ & $0(0)$ & $0(0)$ & $21(100)$ & 14,19 \\
\hline 2006 & 190 & $161(84,74)$ & $1(3,45)$ & $1(3,45)$ & $27(93,10)$ & 15,26 \\
\hline TOTAL & 695 & 83,16 & 1,30 & 0,14 & 15,39 & 16,83 \\
\hline
\end{tabular}

Tabla 3. Causas de profilaxis inadecuada por administración incorrecta

\begin{tabular}{lccc}
\hline & $\begin{array}{c}\text { Elección } \\
\text { de antibiótico } \\
\mathbf{n}(\%)\end{array}$ & $\begin{array}{c}\text { Momento inicio } \\
\text { administración } \\
\mathbf{n}(\%)\end{array}$ & $\begin{array}{c}\text { Duración pauta de } \\
\text { administración } \\
\mathbf{n}(\%)\end{array}$ \\
\hline 2003 & $2(5,13)$ & $32(82,05)$ & $5(12,82)$ \\
2004 & $3(15)$ & $10(50)$ & $7(35)$ \\
2005 & $3(14,28)$ & $16(76,19)$ & $2(9,52)$ \\
2006 & $2(7,40)$ & $24(88,89)$ & $0(0)$ \\
$\mathbf{n}$
\end{tabular}


un porcentaje pequeño de profilaxis inadecuada, y está en relación a la utilización de gentamicina en la biopsia transrectal de próstata y en las vasectomías, siendo el ciprofloxacino el antibiótico de elección en la primera de ellas y no estando protocolizado el administrar antibiótico en las vasectomías.

Hemos de señalar que a lo largo de estos 4 años se han ido desglosando los procedimientos, añadiendo la cirugía genital a partir del 2004 y la cirugía de la incontinencia a partir del año 2006 que antes se incluían en el grupo de otras intervenciones.
Desglosando el cumplimiento de la profilaxis por procedimientos (Fig. 1), observamos que en la cirugía renal y del uréter se observa un cumplimiento con tasas entre el 80 y 94\%, al igual que en la cirugía genital que se sitúa en el $95 \%$ de cumplimiento. En la cirugía endoscópica (sobre todo a expensas de RTU), se observan también tasas altas de cumplimiento (70-81\%).

En la cirugía abierta de próstata se observa un gran aumento en el cumplimiento de la profilaxis pasando del $25 \%$ en 2003 al 78 y $75 \%$ en los años

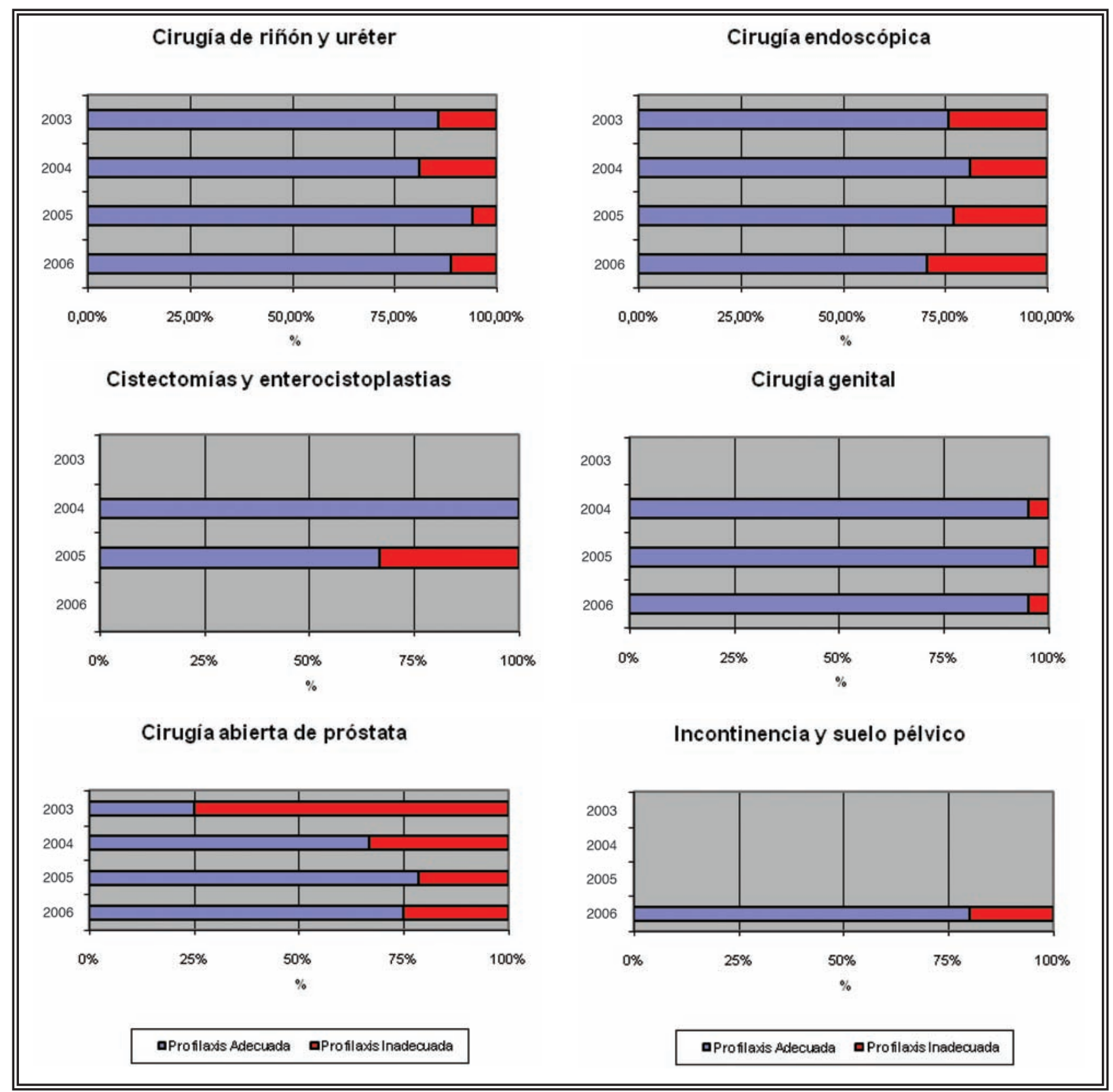

FIGURA 1. Cumplimiento de profilaxis por procedimientos. 
2005 y 2006 respectivamente, debido a las medidas adoptadas ante el bajo cumplimiento detectado al inicio del estudio.

En las cistectomías y/o enterocistoplastias se observa un cumplimiento del 100 y del 66\% en los años 2004 y 2005 respectivamente, pero hay que señalar que sólo se incluyen 3 intervenciones en este periodo.

En otras intervenciones, se han incluido en cada año aquellos procesos no incluidos en los grupos anteriores, con tasas de profilaxis adecuada por encima del 65\% en los 4 años.

\section{DISCUSIÓN}

La profilaxis antibiótica (PA) en los procedimientos quirúrgicos consiste en la administración de antimicrobianos apropiados y a las dosis adecuadas. Su objetivo es disminuir la incidencia de infecciones postoperatorias, sobre todo las de la herida quirúrgica durante el periodo en el que las defensas del individuo no están integras y son más susceptibles a la exposición de agentes patógenos ${ }^{10}$.

En diversos estudios se ha confirmado que el cumplimiento adecuado de las pautas de PA se asocia significativamente a una mayor eficacia, disminuyendo el número de infecciones postoperatorias, la duración y el coste de la estancia ${ }^{2-6,17}$.

Los criterios en la administración de antimicrobianos se apoyan en una serie de puntos aceptados de forma general ${ }^{10}$ :

1. La profilaxis debe aplicarse en cirugías con riego elevado de infección, habiéndose estimado en un $5 \%$ o mayor.

2. El riesgo de infección de cada procedimiento viene determinado por la clasificación de las cirugías de Altemeier, y en función de ésta debe de indicarse la profilaxis. La clasificación de la cirugía transuretral es controvertida, siendo considerada como cirugía limpia-contaminada por los expertos de la EAU ${ }^{17}$.

3. El antibiótico utilizado debe ser eficaz frente al germen más comúnmente implicado en la infección.

4. El antibiótico debe difundir por todos los tejidos afectos por la intervención, alcanzando unos niveles eficaces en los tejidos implicados y sangre durante el procedimiento quirúrgico.

5. Debe de elegirse el antibiótico con mejor relación coste-riesgo/beneficio, siendo el más eficaz, el menos tóxico y utilizado de manera más breve posible ${ }^{18}$.
La vía preferida es la intravenosa, obteniendo unos niveles adecuados en un corto periodo de tiempo. El momento de administración del antibiótico se acepta que es eficaz si disponemos de niveles tisulares suficientes en la zona de incisión al realizar esta, para evitar la contaminación del campo quirúrgico. Otro dato con aceptación unánime es la eficacia de la monodosis profiláctica de antibióticos de vida media larga, pudiendo administrarse unas dosis siguientes si el procedimiento así lo requiere ${ }^{10,12,13,17}$.

El debate sobre el empleo de diferentes pautas antibióticas resulta actualmente contradictorio. Es importante que cada servicio establezca estos protocolos de forma consensuada y uniforme, teniendo en cuenta su idiosincrasia respecto a los procedimientos que realiza, el tipo de paciente y la etiología antimicrobiana, así como una vez aceptados, los ponga en práctica de forma adecuada $7,17,19$.

El no cumplimiento de las pautas recomendadas de PA, así como su aplicación de forma defectuosa conlleva un aumento de costes, así como un aumento de las resistencias bacterianas ${ }^{18}$.

Estos sistemas son una estrategia efectiva del control de costes en el sistema sanitario y repercuten directamente en la mejora de la calidad asistencial que este sistema presta.

La implantación de un sistema de control de cumplimiento de la PA dentro de un hospital constituye un ejercicio multidisciplinar que integra servicios y trabajadores (desde la dirección del hospital, cirujanos, enfermeras y servicios encargados de la vigilancia).

En el momento actual, está fuera de toda duda la eficacia de la PA en cirugía urológica, demostrada en ensayos randomizados controlados. La PA reduce la infección postoperatoria tanto de herida quirúrgica como urinaria ${ }^{10,11,18,21}$.

La elección de los antibióticos de nuestro protocolo se establece en función de los gérmenes más comúnmente aislados. En nuestro hospital, en infección del sitio quirúrgico y en infección urinaria predominan las enterobacterias y Pseudomona Aeruginosa, relegando a los cocos gran positivos a un $2^{\circ}$ lugar $^{10}$. Esto coincide con otras series como la de Johansen et $\mathrm{al}^{20}$.

Revisando la literatura observamos que las pautas administradas son muy variables con respecto a la elección del antibiótico. En cirugía endoscópica las cefalosporinas de $1^{\mathrm{a}}$ y $2^{\mathrm{a}}$ generación son amplia- 
mente utilizadas o recomendas ${ }^{10,13,17}$. Otros autores como Scherz y Larsen postulan, como en nuestro caso, la utilización de una combinación de ampicilina y gentamicina ${ }^{10,13,21}$. Las guías de la EAU no recomienda la profilaxis de forma rutinaria en RTU de tumores vesicales ni en ureteroscopias de litiasis distales. Sin embargo, recomienda su utilización en resecciones de tumores vesicales largas, en RTU de próstata, y en cirugía de litiasis proximales, impactadas o en intervenciones intrarrenales ${ }^{17}$.

En la cirugía del riñón y del uréter, considerada como cirugía limpia en la mayor parte de los casos, hay autores que no la creen necesaria salvo que suponga una cirugía contaminada: nefrolitiasis con apertura de cavidades, cirugías muy prolongadas o apertura de otras cavidades durante el trascurso de la intervención ${ }^{10,17}$. En nuestro caso, utilizamos amoxicilina-clavulánico de forma sistemática y está aprobado por la Comisión de Infecciones del hospital.

En la cistectomía con neovejiga la eficacia está probada y las pautas son diversas, incluyendo combinaciones de clindamicina o metronidazol junto a gentamicina, cefalosporinas de $3^{a}$ generación con penicilina-metronidazol ${ }^{10,17,21}$. En nuestro hospital utilizamos una triple terapia durante 5 días debido a la alta tasa de infección de herida por Escherichia Coli, que hemos presentado en los años previos.

La cirugía abierta de próstata supone de forma general una cirugía limpia-contaminada, utilizando la amoxicilina clavulánico si el paciente no tiene sonda. En pacientes con sonda, asumimos que se convierte en una cirugía contaminada, con mayor tasa de IQ, por lo que administramos una profilaxis prolongada que combina ampicilina y gentamicina para cubrir Enterococcus faecalis, Escherichia Coli y Pseudomona Aeruginosa, gérmenes más frecuentemente implicados. La guía de la EAU recomienda la profilaxis rutinaria con cefalosporinas de $2^{\mathrm{a}}$ o $3^{\mathrm{a}}$ generación, cotrimoxazol o aminopenicilinas, sin diferenciar ambos grupos ${ }^{17}$.

En la cirugía genital, exceptuando la circuncisión, vasectomía e intervenciones sobre el frenillo en las que no se utiliza profilaxis, utilizamos de rutina la amoxicilina-clavulánico, a pesar de que está establecido de que no es necesario al ser cirugía limpia (clase I) 10,17,21.

Esto nos hace pensar que dada la baja tasa de IQ que tenemos, la profilaxis en esta cirugía se podría suprimir.
En la cirugía protésica coincidimos con la mayor parte de los autores en la utilización de vancomicina asociada o no a gentamicina ${ }^{10,21,23}$. La guía de la EAU establece las cefalosporinas de $2^{\mathrm{a}}$ y $3^{\mathrm{a}}$ generación o penicilinas como recomendación en este tipo de cirugía $^{17}$.

La falta de cumplimiento de estas pautas conlleva una serie de consecuencias: Una de ellas es el coste, tanto a la hora de prolongar una terapia innecesaria, como en la aparición de una infección nosocomial. Otro factor es el aumento de las resistencias bacterianas al realizar profilaxis prolongadas. Por último la aparición de infección nosocomial conlleva un aumento de la estancia hospitalaria, en las complicaciones y en el bienestar del paciente $^{2-6,18}$.

Concretando en nuestra revisión, hemos de destacar que el cumplimiento de PA en nuestro servicio es alto, por encima de los estándares fijados como cumplimiento adecuado.

Sin embargo, no existen en la literatura muchos artículos sobre los porcentajes de cumplimiento de PA en Urología. Cano presenta un cumplimiento global del 57\% y del 77\% en una comparación de 2 protocolos antibióticos aplicados en el servicio de Urología de su hospital ${ }^{19}$.

Observando la profilaxis inadecuada, en nuestro estudio, se observa que es la pauta administrada estando indicada pero de forma incorrecta, la que constituye el grupo más frecuente.

Dentro de esta pauta incorrecta, se observa que el mayor porcentaje de errores se debe al retraso en el momento de la administración del antibiótico, lo que conlleva una alta probabilidad de que los niveles tisulares del mismo no sean los adecuados en el momento en que se inicia el procedimiento quirúrgico, por no haber dado tiempo suficiente a que este difunda. Para que una profilaxis antibiótica sea efectiva, necesitamos niveles tisulares suficientes en el momento de la incisión, para evitar la contaminación de la zona operatoria, precursor necesario para que se produzca una IQ. En un segundo lugar la duración incorrecta de la pauta antibiótica, debida fundamentalmente a la omisión de las dosis postoperatorias en cirugía abierta de próstata con sonda, y por la prolongación no indicada en la cirugía renal y del uréter por desconfianza en la dosis única de antibiótico. 
En nuestro servicio, el retraso en el momento de la administración del antibiótico se debe a problemas estructurales del hospital en los últimos años en el que el paciente baja directamente a quirófano, donde el anestesista administra el antibiótico una vez terminado el proceso anestésico, y no en la inducción como está indicado. Este problema se hace más patente en las pautas que constan de más de un antibiótico (cirugía endoscópica y cirugía abierta de próstata con sonda), así como en las cirugías en las que el tiempo de intervención es corto (por ejemplo en intervenciones realizadas bajo raquianestesia). Se están haciendo intentos de coordinar esta actuación, hasta que solucionemos el problema estructural de disponer de una sala de pre-anestesia donde se pueda comenzar a administrar el antibiótico.

Cano en su revisión presenta el mantenimiento excesivo de la pauta antibiótica como la causa más frecuente de su profilaxis inadecuada, mientras que la elección inadecuada del antibiótico y la omisión de la dosis preoperatoria se encuentran por detrás ${ }^{15}$.

Analizando por procedimientos, observamos un cumplimiento alto en la cirugía renal y de uréter, la cirugía genital y la cirugía endoscópica, todas ellas con una tasa de cumplimiento por encima del 70\%. Señalar que se observa un leve aumento en la profilaxis inadecuada en la cirugía endoscópica, en relación a la administración tardía del antibiótico, antes señalada.

En las cistectomías y/o enterocistoplastias presentamos el cumplimiento de los años 2004 y 2005 que consideramos adecuado, señalando que el volumen de intervenciones en este grupo es escaso, sin poder extraer conclusiones significativas por el pequeño tamaño muestral.

Es en la cirugía abierta de próstata donde más se observa la eficacia de la evaluación periódica del protocolo de PA. En 2003 presentamos una tasa de cumplimiento del 25\%, muy baja en comparación al estándar. Este bajo cumplimiento estaba en relación a la falta de administración de las 3 dosis postoperatorias de ampicilina en los pacientes portadores de sonda, y en menor medida a la elección inadecuada del antibiótico. Tras las medidas propuestas, revisando y actualizando los protocolos dentro de un entorno multidisciplinar, se ha conseguido un gran aumento del cumplimiento en este proceso, llegando a valores por encima del 70\%.
En el grupo de otras intervenciones, el cumplimiento de profilaxis se sitúa por encima del 60\%. En este grupo se incluyen el resto de procedimientos que no tienen cabida en otros grupos. Esto junto a la creación en el transcurso del estudio de nuevos grupos como son el de cirugía genital y de cirugía de la incontinencia hacen difícil su comparación con otros servicios de Urología.

Por tanto, debemos señalar la importancia del establecimiento de protocolos antibióticos adecuados a los gérmenes más frecuentemente implicados en las infecciones nosocomiales post-quirúrgi$\operatorname{cas}^{20}$. Para esto es necesario la realización de estudios periódicos de la flora responsable de las infecciones quirúrgicas urológicas en cada centro para detectar cambios en los patógenos más habituales, así como en las resistencias a los antimicrobianos $^{10}$.

Este estudio pone de manifiesto la importancia, ya conocida, de la revisión continuada de los protocolos de PA así como la realización de una evaluación periódica de los mismos, para concienciar a los profesionales en la unificación de criterios y aumentar su adhesión al mismo $7,10,17,19,24,25$.

\section{CONCLUSIÓN}

La efectividad de la PA requiere la existencia de protocolos adecuados y actualizados para la unificación de criterios entre los profesionales, para detectar nuevos problemas, así como para buscar soluciones para un correcto cumplimiento del mismo.

La vigilancia del incumplimiento de los protocolos no debe quedarse en una evaluación de los resultados, sino que se debe analizar la estructura y el proceso de la práctica clínica. De este análisis se pueden obtener conclusiones y medidas prácticas para resolver los problemas reflejados en la evaluación.

Es fundamental que en este proceso de evaluación estén implicados todas las personas que colaboran en su funcionamiento: urólogos, anestesistas, enfermeras, personal del servicio de Medicina Preventiva y la Comisión de Infecciones del hospital. Esta es la manera de obtener acuerdos y medidas de actuación práctica, aceptadas por todos los grupos, que se reflejen en un aumento de la adhesión al cumplimiento del protocolo antibiótico. 


\section{REFERENCIAS}

1. Mangram AJ, Horan TC, Pearson ML, Silver LC, Jarvis WR. Guideline for prevention of surgical site infection, 1999. Hospital Infection Control Practices Advisory Committee. Infection Control and Hospital Epidemiology. 1999;20(4):250279

2. Haley RW, Schaberg DR, Crossley KB, Von Allmen SD, McGowan JE Jr. Extra charges and prolongation of stay attributable to nosocomial infections: A prospective interhospital comparison. Am J of Med. 1981;70(1):51-58.

3. Moris de la Tassa J, Fernández Muñoz P, Antuña Egocheaga A, Gutiérrez del Río MC, Cartón Sánchez JA. Estimación de los costes asociados con la infección del tracto urinario. Un estudio de casos y controles. Rev Clin Esp. 2003;203(3):119-124.

4. Coello R, Glenister H, Fereres J, Bartlett C, Leigh D, Sedgwick $J$, et al. The cost of infection in surgical patients: a case-control study. J Hosp Infect. 1993;25(4):239-250.

5. Asensio Vegas A, Monge Jodrá y Lizán García M. Nosocomial infection in surgical wards: a controlled study of increased duration of hospital stays and direct cost of hospitalization. Europ J Epidemiol. 1993;5:504-510.

6. Lizán-García M, Gallego C, Martínez I. La infección de localización quirúrgica: una aproximación al coste atribuible. Medicina Preventiva 2004; vol X: 6-11.

7. Sanz C, Fernandez J, Sáinz A, Moral L. Evaluación de los protocolos de antibioprofilaxis quirúrgica en los hospitales del INSALUD. Medicina Preventiva VI (3) 2000; 23-27.

8. Alianza Mundial para la Seguridad del paciente. Organización Mundial de la Salud. Octubre 2004. http://www.who.int/ patientsafety/en/

9. Plan de calidad para el Sistema nacional de Salud. Marzo 2006. Ministerio de Salud y Consumo. http://www.msc.es/organizacion/sns/planCalidadSNS/home.htm

10. Llanes González L, Ruiz de la Roja JC, Martín Oses E, de Paz Cruz L, Zarate Rodríguez E, Sánchez Sánchez E, et al. Profilaxis antimicrobiana en Urología. Actas Urol Esp. 1997;21(6): 540-548.

11. Hargreave TB, Botto H, Rikken GH, Hindmarsh JR, McDermott TE, Mjolnerod OK, et al. European Collaborative Study of Antibiotic Prophylaxis for Transurethral Resection of the Prostate. Eur Urol. 1993;23(4):437-443.

12. Del Rio G, Dalet F, Chechile G. Antimicrobial prophylaxis in urologic surgery: does it give some benefit?. Eur Urol. 1993;24 (3):305-312.

13. Scherz H.C, Lowell C. Prophylactic Antibiotics in Urology. Urol Clin North Am. 1987;14(2):265-271.

14. Aldana Espinal JM, Valencia R, Gasch A, Conde M, Flores JM, Murillo F. La infección nosocomial como indicador de calidad asistencial. Comunicación en el XII Congreso de la Sociedad Española de Medicina Preventiva, Salud Pública e Higiene.
15. Ballestero Diego R, Rebollo Rodrigo H, Gutiérrez Baños J.L, Aguilera Tubet C, Zubillaga Guerrero S, Martín García B. Infección nosocomial y del sitio quirúrgico en un hospital de tercer nivel (2002-2005). Actas Urol Esp. 2006;30(9):905- 912.

16. Vaqué J. y grupo de trabajo EPINE: Evolución de la prevalencia de las infecciones nosocomiales en los hospitales españoles. EPINE 1990-2003. DL: M 36810-2004:176-177.

17. Naber K.G, Bishop M.C, Bjerklund-Johansen T.E, Botto H, Çek M, Grabe M, Lobel B, Palou J, Tenke P. Guidelines on the management of urinary and male genital tract infection. 2006. http://www.uroweb.org/nc/professionalresources /guidelines/online/

18. Delgado Rodriguez M. Quimioprofilaxis en cirugía: Problemas en su cumplimiento. Ponencia en las I Jornadas Internacionales y IV Nacionales sobre avances en Medicina Preventiva. Marzo 2001:69-76.

19. Cano M, Castrodeza J, Fernández E, De la Cruz M. Utilización de dos protocolos de profilaxis antibiótica en el servicio de Urología del H.C.U. de Valladolid. Medicina Preventiva vol.VI (1) 2000:12-18.

20. Bjerklund Johansen T.E, Cek M, Naber K, Stratchounski L, Svendsen M.V, Tenke P. Prevalence of Hospital-Acquired Urinary Tract Infections in Urology Departments. Eur Urol. 2007;51(4):1100-1111.

21. Larsen EH, Gasser TC, Madsen PO. Antimicrobial Prophylaxis in Urologic Surgery. Urol Clin North Am. 1986;13(4):591-601.

22. Grace M. Controversies in antibiotic prophylaxis in urology. Int J Antimicrob Agents 2004;23Suppl 1:S17-23.

23. Calcerrada Diaz-Santos N, Villena Ugarte E, Sánchez Blanque R, Asúnsolo del Barco A, Jadraque Jiménez P, Troncoso Viejo, D. Rincón Carlavilla A, Silveira Guijarro L. Evaluación de la profilaxis quirúrgica adecuada a protocolo. Comunicación en las I Jornadas Internacionales y IV Nacionales sobre avances en Medicina Preventiva. Marzo 2001:159-160. Importancia cumplimiento.

24. Alerany C, Campany D, Monterde J, Semeraro C. Impact of local guidelines and an integrated dispensing system on antibiotic prophylaxis quality in a surgical centre. J Hosp Infect $J$ Hosp Infect. 2005;60(2):111-117.

25. Gomez MI, Acosta-Gnass SI, Mosqueda-Barboza L, Basualdo JA. Reduction in surgical antibiotic prophylaxis expenditure and the rate of surgical site infection by means of a protocol that controls the use of prophylaxis. Infect Control Hosp. Epidemiol. 2006;27(12):1358-1365.

Correspondencia autor: Dr. R. Ballestero Diego

Servicio de Urología. Hospital Univ. Marqués de Valdecilla Avda. de Valdecilla, 25 - 39008 Santander (Cantabria)

Tel.: 942202520

E-mail autor: badiro2@yahoo.es

Información artículo: Original - Infección urinaria

Trabajo recibido: noviembre 2007

Trabajo aceptado: enero 2008 